\title{
Masses and other parameters of massive binaries
}

\author{
Douglas R. Gies \\ Center for High Angular Resolution Astronomy, Department of Physics \\ and Astronomy, Georgia State University, Atlanta, GA 30303, USA
}

\begin{abstract}
Binary stars provide us with the means to measure stellar mass. Here I present several lists of known O-type stars with reliable mass estimates that are members of eclipsing, double-lined spectroscopic binaries. The masses of young, unevolved stars in binaries are suitable for testing the predictions of evolutionary codes, and there is good agreement between the observed and predicted masses (based upon temperature and luminosity) if the lower temperature scale from line-blanketed model atmospheres is adopted. A final table lists masses for systems in a wide variety of advanced evolutionary stages.
\end{abstract}

\section{Introduction}

There are two direct techniques for measuring stellar mass. The first is based upon radial velocity curves of binaries where the spectral lines of both components are readily observable. The orbital elements for such double-lined spectroscopic binaries (SB2) yield $M \sin ^{3} i$ for each star, but we need to determine the orbital inclination $i$ in order to estimate the mass $M$. If our line of sight lies close to the orbital plane and the stars display mutually eclipses, then the inclination and stellar radii $R$ can be determined by fitting the photometric light curve. Binaries with separations small compared to their radii will be tidally distorted, and the resulting 'ellipsoidal' light variations can also be used to find the orbital inclination (with lower precision because the light curve amplitude depends on the inclination and the estimated stellar radii). Analyses of combined spectroscopic and photometric data are generally restricted to short period binaries because the probability of eclipses decreases with increasing separation. However, the masses of close binaries may be altered by mass transfer or binary-related mass loss, so we need to restrict our sample to unevolved binaries when comparing the observed masses with predictions for single stars. The second method is based upon the measurement of the astrometric orbit through high angular resolution techniques. The total system mass can be derived from the angular orbit and distance, but if radial velocity curves are available then the individual masses follow as well as an independent estimate of the distance. We are entering a new era of high angular resolution astronomy using optical interferometers, and we can anticipate some exciting observations of the small angular orbits of massive binaries. An example is the long period binary $15 \mathrm{Mon}$ (Gies et al. 1993, 1997), the first massive system discovered to fall in the period range suitable for spectroscopic and astrometric study.

There are a number of indirect techniques for determining stellar mass involving apsidal motion in binaries (Benvenuto et al. 2002), placement in the $\mathrm{H}$ - 
$\mathrm{R}$ diagram with respect to evolutionary models (Burkholder, Massey \& Morrell 1997), and spectroscopic diagnostics of the photosphere and wind (Herrero, Puls $\&$ Villamariz 2000). We can use the results from direct methods to test the validity of the indirect schemes, and study, for example, the mass discrepancy between evolutionary and spectroscopic masses (Herrero et al. 1992). This review extends work by Batten (1968), Hutchings (1975), Popper (1980), Hilditch \& Bell (1987), Andersen (1991), Figueiredo, De Greve \& Hilditch (1994), Schönberner \& Harmanec (1995), Harries \& Hilditch (1998a), and Ribas et al. (2000).

\section{Unevolved stars in binaries}

Table 1 lists data on 12 important SB2 systems containing unevolved stars. The columns give the name of the system and the spectral classification, mass, effective temperature, radius, and equatorial rotational velocity for the primary and secondary, respectively. The final column gives a reference code based on the first three letters of the leading author's name and last two digits of the publication date (repeated in the reference list). Generally only one reference is given, but there are several cases ( $Y$ Cyg, DH Cep) where I give the results from multiple studies which display good consistency. The record holder in this group is $\mathrm{R} 136-38$ which resides in the $\mathrm{R} 136$ cluster in the Large Magellanic Cloud. Massey, Penny \& Vukovich (2002) estimate that the O3V primary in $\mathrm{R} 136-38$ has a mass of $56.9 \pm 0.6 \mathrm{M}_{\odot}$. This is the most reliable estimate of mass among the candidates for the most massive star ever measured (Plaskett's star: $\gtrsim 48 \pm 5 \mathrm{M}_{\odot}$, Bagnuolo, Gies \& Wiggs 1992; WR 47: 60 $\mathrm{M}_{\odot}$, Lamontagne et al. 1996; WR 22: 55.3 $\pm 7.3 \mathrm{M}_{\odot}$, Schweickhardt et al. 1999).

I used the values of $T_{\text {eff }}$ and $R$ given in Table 1 to determine the predicted mass by interpolating in $\left(\log T_{\text {eff }}, \log L / L_{\odot}\right)$ plane within the evolutionary grids of Schaller et al. (1992; $Z=0.020$ for Galactic stars) and Schaerer et al. (1993; $Z=0.008$ for LMC stars). The resulting evolutionary masses are plotted against the observed masses in Figure 1a. The agreement is reasonable but there is an uncomfortable trend for the evolutionary masses to be larger than the observed ones. Harries \& Hilditch (1998a) suggested that residual problems with the adopted temperature scale for $\mathrm{O}$-stars could explain this trend. Martins, Schaerer, Hillier (2002) demonstrated that hitherto neglected sources of line blanketing tend to reduce the effective temperatures associated with any given spectral subtype, and so I repeated the calculation of evolutionary masses using their spectral type $-T_{\text {eff }}$ relation for dwarf stars. The resulting better agreement between the evolutionary and observed masses (Figure 1b) indicates that the evolutionary models are fully consistent with the available data. Recent models by Meynet \& Maeder (2000) and Heger \& Langer (2000) show how rapid rotation can alter a star's evolutionary track in the H-R diagram, so a more careful comparison should account for the rotational velocities given in Table 1. Table 2 lists nine other non-eclipsing systems with $M \sin ^{3} i>10 \mathrm{M}_{\odot}$ that generally have lower mass limits which are consistent with the data in Table 1.

\section{Evolved stars in binaries}

Table 3 lists mass estimates for a sample of 30 binaries that contain evolved Otype stars (for a list of WR+O systems, see van der Hucht 2001). I attempted to 
Table 1. Young detached binary systems (primary/secondary).

\begin{tabular}{|c|c|c|c|c|c|c|}
\hline $\begin{array}{l}\text { name/ } \\
\text { HD/other }\end{array}$ & $\begin{array}{l}\text { spectral } \\
\text { type }\end{array}$ & $\begin{array}{l}M \\
\left(\mathrm{M}_{\odot}\right)\end{array}$ & $\begin{array}{l}T_{\text {eff }} \\
(\mathrm{kK})\end{array}$ & $\begin{array}{l}R \\
\left(\mathrm{R}_{\odot}\right)\end{array}$ & $\begin{array}{l}v_{\mathrm{eq}} \\
\left(\mathrm{km} \mathrm{s}^{-1}\right)\end{array}$ & Ref. \\
\hline $\begin{array}{l}\text { R 136-38 } \\
\text { (LMC) }\end{array}$ & $\begin{array}{l}\mathrm{O} 3 \mathrm{~V} \\
\mathrm{O} 6 \mathrm{~V}\end{array}$ & $\begin{array}{l}56.9(6) \\
23.4(2)\end{array}$ & $\begin{array}{l}48.5(49) \\
42.2(42)\end{array}$ & $\begin{array}{l}9.3(10) \\
6.4(7)\end{array}$ & $\begin{array}{c}132(20) \\
92(20)\end{array}$ & MAS02 \\
\hline $\begin{array}{l}\text { R } 136-42 \\
\text { (LMC) }\end{array}$ & $\begin{array}{l}\mathrm{O} 3 \mathrm{~V} \\
\mathrm{O} 3 \mathrm{~V}\end{array}$ & $\begin{array}{l}40.3(1) \\
32.6(1)\end{array}$ & $\begin{array}{l}48.5(49) \\
48.5(49)\end{array}$ & $\begin{array}{l}7.4(8) \\
6.7(7)\end{array}$ & $\begin{array}{l}100(20) \\
130(30)\end{array}$ & MAS02 \\
\hline $\begin{array}{l}\text { R136-77 } \\
\text { (LMC) }\end{array}$ & $\begin{array}{l}05.5 \mathrm{~V} \\
\text { O5.5 V }\end{array}$ & $\begin{array}{l}28.9(3) \\
26.2(3)\end{array}$ & $\begin{array}{l}43.2(43) \\
43.2(43)\end{array}$ & $\begin{array}{l}5.8(5) \\
5.8(5)\end{array}$ & $\begin{array}{l}141(20) \\
131(20)\end{array}$ & MAS02 \\
\hline CPD $-59^{\circ} 2628$ & $\begin{array}{l}09.5 \mathrm{~V} \\
\text { B0.3 V }\end{array}$ & $\begin{array}{l}14.0(20) \\
11.7(18)\end{array}$ & $\begin{array}{l}32.0(5) \\
30.0(8)\end{array}$ & $\begin{array}{l}5.3(3) \\
4.4(2)\end{array}$ & $\begin{array}{l}172(10) \\
152(10)\end{array}$ & FRE01 \\
\hline $\mathrm{CPD}-59^{\circ} 2603$ & $\begin{array}{l}\text { O7 V } \\
\text { O9.5 V }\end{array}$ & $\begin{array}{l}22.7 \\
14.5\end{array}$ & $\begin{array}{l}37.5(15) \\
32.8(13)\end{array}$ & $\begin{array}{l}7.1(9) \\
4.9(5)\end{array}$ & $\begin{array}{l}167 \\
116\end{array}$ & RAU01 \\
\hline \multirow[t]{2}{*}{$\begin{array}{l}\text { EM Car } \\
\text { HD } 97484\end{array}$} & $\begin{array}{l}08 \mathrm{~V} \\
08 \mathrm{~V}\end{array}$ & $\begin{array}{l}22.9(3) \\
21.4(3)\end{array}$ & $\begin{array}{l}34.0(20) \\
34.0(20)\end{array}$ & $\begin{array}{l}9.3(2) \\
8.3(2)\end{array}$ & $\begin{array}{l}152(20) \\
131(15)\end{array}$ & AND89 \\
\hline & $\begin{array}{l}\mathrm{O} 8 \mathrm{~V} \\
\mathrm{O} 8 \mathrm{~V}\end{array}$ & $\begin{array}{l}22.3 \\
20.3\end{array}$ & $\begin{array}{l}\ldots \\
\ldots\end{array}$ & $\begin{array}{l}\ldots \\
\ldots\end{array}$ & $\begin{array}{l}145(10) \\
135(9)\end{array}$ & STI94a \\
\hline $\begin{array}{l}\text { V1036 Sco } \\
\text { HD } 159176\end{array}$ & $\begin{array}{l}\text { O6 V } \\
\text { O7 V }\end{array}$ & $\begin{array}{l}\text { 32. (4) } \\
\text { 32. (4) }\end{array}$ & $\begin{array}{l}42.5 \\
35.0\end{array}$ & $\begin{array}{l}9.4(8) \\
9.4(8)\end{array}$ & $\begin{array}{l}196(20) \\
196(20)\end{array}$ & PAC96 \\
\hline $\begin{array}{l}\text { V3903 Sgr } \\
\text { HD } 165921\end{array}$ & $\begin{array}{l}\text { O7 V } \\
\text { O9 V }\end{array}$ & $\begin{array}{l}27.3(6) \\
19.0(4)\end{array}$ & $\begin{array}{l}38.0(19) \\
34.1(17)\end{array}$ & $\begin{array}{l}8.09(9) \\
6.13(6)\end{array}$ & $\begin{array}{l}253(25) \\
187(19)\end{array}$ & VAZ97 \\
\hline $\begin{array}{l}\text { V1182 Aql } \\
\text { HD } 175514\end{array}$ & $\begin{array}{l}\mathrm{O} 8 \mathrm{~V} \\
\mathrm{~B} 1 \mathrm{~V}\end{array}$ & $\begin{array}{l}37.8(16) \\
13.5(5)\end{array}$ & $\begin{array}{l}35.6(15) \\
27.5(15)\end{array}$ & $\begin{array}{l}8.8(2) \\
5.9(1)\end{array}$ & $\begin{array}{l}\ldots \\
\ldots\end{array}$ & BEL87 \\
\hline $\begin{array}{l}\text { V478 Cyg } \\
\text { HD } 193611\end{array}$ & $\begin{array}{l}09.5 \mathrm{~V} \\
09.5 \mathrm{~V}\end{array}$ & $\begin{array}{l}16.6(9) \\
16.3(9)\end{array}$ & $\begin{array}{l}30.5(11) \\
30.5(11)\end{array}$ & $\begin{array}{l}7.4(1) \\
7.4(1)\end{array}$ & $\begin{array}{l}\ldots \\
\ldots\end{array}$ & POP91 \\
\hline \multirow[t]{3}{*}{$\begin{array}{l}\text { Y Cyg } \\
\text { HD } 198846\end{array}$} & $\begin{array}{l}\text { O9.8 } \\
\text { O9.8 }\end{array}$ & $\begin{array}{l}17.6(3) \\
17.0(3)\end{array}$ & $\begin{array}{l}34.5(6) \\
34.2(6)\end{array}$ & $\begin{array}{l}5.93(7) \\
5.78(7)\end{array}$ & $\begin{array}{l}142(2) \\
138(2)\end{array}$ & SIM94 \\
\hline & $\begin{array}{l}\text { O9.3 } \\
\text { O9.4 }\end{array}$ & $\begin{array}{l}17.5(4) \\
17.3(3)\end{array}$ & $\begin{array}{l}31.0(20) \\
31.6(20)\end{array}$ & $\begin{array}{l}6.0(3) \\
5.7(3)\end{array}$ & $\begin{array}{l}147(10) \\
138(10)\end{array}$ & HIL95 \\
\hline & $\begin{array}{l}09 \mathrm{~V} \\
09.5 \mathrm{~V}\end{array}$ & $\begin{array}{l}16.8(5) \\
17.7(4)\end{array}$ & $\begin{array}{l}35.9(21) \\
34.9(10)\end{array}$ & $\begin{array}{l}8.4(28) \\
7.7(25)\end{array}$ & $\begin{array}{l}\ldots \\
\ldots\end{array}$ & BUR97 \\
\hline \multirow[t]{4}{*}{$\begin{array}{l}\text { DH Cep } \\
\text { HD } 215835\end{array}$} & $\begin{array}{l}\ldots \\
\ldots\end{array}$ & $\begin{array}{l}29.4(4) \\
25.0(3)\end{array}$ & $\begin{array}{l}44.0(10) \\
43.0(10)\end{array}$ & $\begin{array}{l}8.31(6) \\
7.76(6)\end{array}$ & $\begin{array}{l}263(20) \\
247(20)\end{array}$ & STU94 \\
\hline & $\begin{array}{l}\mathrm{O} 5.5 \mathrm{~V} \\
\mathrm{O} 6.5 \mathrm{~V}\end{array}$ & $\begin{array}{l}32.7(17) \\
29.8(16)\end{array}$ & $\begin{array}{l}41.0(20) \\
39.6(20)\end{array}$ & $\begin{array}{l}8.7(3) \\
8.6(3)\end{array}$ & $\begin{array}{l}\ldots \\
\ldots\end{array}$ & HIL96 \\
\hline & $\begin{array}{l}\mathrm{O} 6 \mathrm{~V} \\
\mathrm{O} 7 \mathrm{~V}\end{array}$ & $\begin{array}{l}41(4) \\
34(3)\end{array}$ & $\begin{array}{l}42.0(30) \\
39.0(15)\end{array}$ & $\begin{array}{l}10.2(6) \\
9.7(6)\end{array}$ & $\begin{array}{l}258(10) \\
226(9)\end{array}$ & PEN97 \\
\hline & $\begin{array}{l}\text { O5.5 III(f) } \\
\text { O6 III(f) }\end{array}$ & $\begin{array}{l}34.4(27) \\
29.8(25)\end{array}$ & $\begin{array}{l}41.3(20) \\
40.2(20)\end{array}$ & $\begin{array}{l}13.9(13) \\
13.4(12)\end{array}$ & $\begin{array}{l}\ldots \\
\ldots\end{array}$ & BUR97 \\
\hline
\end{tabular}

Note: numbers in parentheses give the error in the last digit quoted.

group these according to evolutionary status (Vanbeveren, van Rensbergen \& de Loore 1998), but in many cases the evolutionary status is open to debate. The first group represents an early stage where the initially more massive primary has evolved away from the main sequence but has not suffered large scale mass

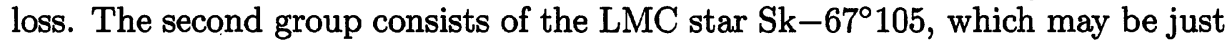
beginning mass transfer, and two short-period contact systems. The third and largest group represents the post-mass transfer ('Algol') stage where we find a semi-detached system consisting of a hot, massive gainer and a cool, evolved 

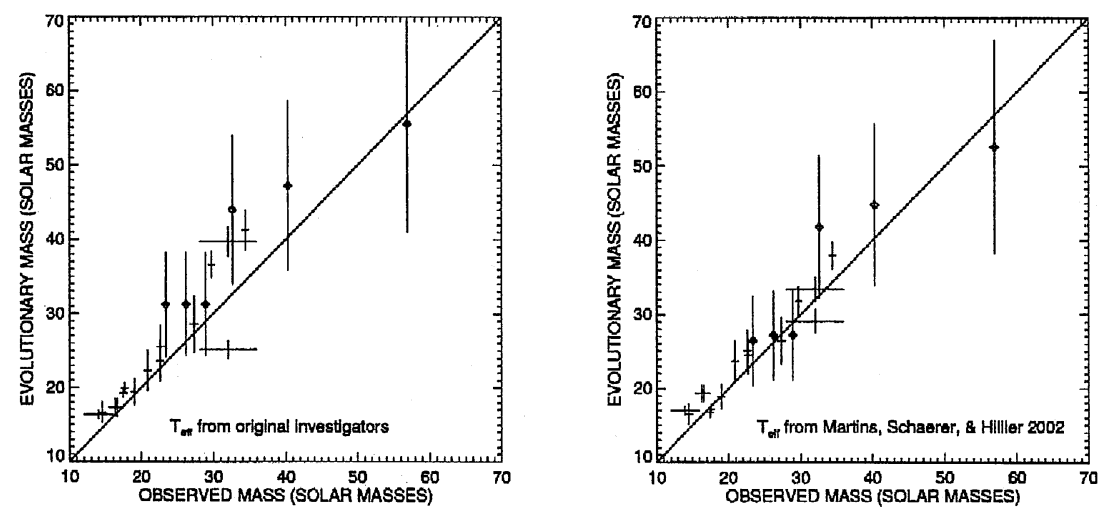

Figure 1. Left: A comparison of the observed and calculated evolutionary masses of $\mathrm{O}$-stars in binaries made using the temperatures and radii given in the papers cited in Table 1. Diamonds indicate stars in the LMC. Right: A similar comparison but with evolutionary masses calculated using the lower temperature scale advocated by Martins et al. (2002).

Table 2. Non-eclipsing young detached binary systems (primary/secondary).

\begin{tabular}{lllllll}
\hline \hline $\begin{array}{l}\text { name/ } \\
\text { HD/other }\end{array}$ & $\begin{array}{l}\text { spectral } \\
\text { type }\end{array}$ & $\begin{array}{l}M> \\
\left(\mathrm{M}_{\odot}\right)\end{array}$ & $\begin{array}{l}T_{\text {eff }} \\
(\mathrm{kK})\end{array}$ & $\begin{array}{l}R \\
\left(\mathrm{R}_{\odot}\right)\end{array}$ & $\begin{array}{l}v_{\text {eq }}> \\
\left(\mathrm{km} \mathrm{s}^{-1}\right)\end{array}$ & Ref. \\
\hline R136-39 & O3 V & 27.2 & $48.5(49)$ & $8.1(6)$ & $\ldots$ & MAS02 \\
(LMC) & O5.5 V & 20.5 & $43.2(43)$ & $7.1(6)$ & $\ldots$ & \\
ST 1-98 & O4 V & 45. & $\ldots$ & 12. & $\ldots$ & BER98 \\
(LMC) & O4 V & 45. & $\ldots$ & 12. & $\ldots$ & \\
HD 75759 & O9 V & 17.8 & $\ldots$ & $\ldots$ & $\ldots$ & THA66 \\
& O9 V & 14.3 & $\ldots$ & $\ldots$ & $\ldots$ & \\
HD 93205 & O3 V & $33.0(16)$ & 51.3 & $\ldots$ & $\ldots$ & MOR01 \\
& O8 V & $15.2(15)$ & 38.0 & $\ldots$ & $\ldots$ & ANT00 \\
CPD -59 2635 & O8 V & 15.5 & 38.5 & $9.1(4)$ & $\ldots$ & ALB01 \\
& O9.5 V & 11.4 & 34.6 & $6.5(5)$ & $\ldots$ & \\
HD 101131 & O6.5 V((f)) & $25.3(25)$ & $40.5(15)$ & $9.5(9)$ & $102(10)$ & GIE02 \\
& O8.5 V & $14.1(15)$ & $35.0(15)$ & $7.7(8)$ & $164(12)$ & \\
HD 152218 & O9.5 IV & $14.2(6)$ & 31.6 & 11.2 & $152(10)$ & STI97a \\
& O9.5 V & $10.6(5)$ & 31.6 & 7.9 & $133(30)$ & \\
& O9 IV & $13.6(13)$ & $\ldots$ & $\ldots$ & $\ldots$ & GAR01 \\
& $\ldots$ & $12.6(17)$ & $\ldots$ & $\ldots$ & $\ldots$ & \\
HD 191201 & O9.5 V-III & $12.9(29)$ & $33.9(7)$ & $13.0(13)$ & $\ldots$ & BUR97 \\
& O9.5 V-III & $11.0(9)$ & $33.9(7)$ & $9.9(9)$ & $\ldots$ & \\
& $\ldots$ & $12.8(6)$ & $\ldots$ & $\ldots$ & $\ldots$ & STI01 \\
& $\ldots$ & $12.2(5)$ & $\ldots$ & $\ldots$ & $\ldots$ & \\
HD 206267 & O5 V & $22.1(25)$ & $\ldots$ & $\ldots$ & $\ldots$ & STI95a \\
& O7/8 V & $12.3(8)$ & $\ldots$ & $\ldots$ & $\ldots$ & \\
& O6.5 V((f)) & $28.4(15)$ & $41.2(10)$ & $13.9(26)$ & $\ldots$ & BUR97 \\
& O9.5: V & $17.3(15)$ & $4.9(10)$ & $8.9(16)$ & $\ldots$ & \\
\hline \hline
\end{tabular}


Table 3. Evolved systems (primary/secondary).

\begin{tabular}{|c|c|c|c|c|c|c|}
\hline $\begin{array}{l}\text { name/ } \\
\text { HD/other }\end{array}$ & $\begin{array}{l}\text { spectral } \\
\text { type }\end{array}$ & $\begin{array}{l}M \\
\left(\mathrm{M}_{\odot}\right) \\
\end{array}$ & $\begin{array}{l}T_{\text {eff }} \\
(\mathrm{kK})\end{array}$ & $\begin{array}{l}R \\
\left(\mathrm{R}_{\odot}\right)\end{array}$ & $\begin{array}{l}v_{\mathrm{eq}} \\
\left(\mathrm{km} \mathrm{s}^{-1}\right)\end{array}$ & Ref. \\
\hline \multicolumn{7}{|c|}{ before interaction stage } \\
\hline $\begin{array}{l}\text { CC Cas } \\
\text { HD } 19820\end{array}$ & $\begin{array}{l}\text { O8.5 III } \\
\text { B0.5 V }\end{array}$ & $\begin{array}{l}18.3(5) \\
7.6(2)\end{array}$ & $\begin{array}{l}34.5(20) \\
28.3(20)\end{array}$ & $\begin{array}{l}10.08(14) \\
4.02(23)\end{array}$ & $\begin{array}{l}165(5) \\
<100\end{array}$ & HIL94 \\
\hline $\begin{array}{c}05: 34: 41.3 \\
-69: 31: 39\end{array}$ & $\begin{array}{l}\text { O3 If* } \\
\text { O6: V }\end{array}$ & $\begin{array}{l}41.2(12) \\
27.0(12)\end{array}$ & $\begin{array}{l}50.0 \\
47.2(17)\end{array}$ & $\begin{array}{l}9.56(2) \\
7.99(5)\end{array}$ & $\begin{array}{l}\ldots \\
\cdots\end{array}$ & OST01a \\
\hline $\begin{array}{l}\text { KX Vel } \\
\text { HD 75821 }\end{array}$ & $\begin{array}{l}\text { O9.5 II } \\
\ldots\end{array}$ & $\begin{array}{l}>14.5 \\
>8.4\end{array}$ & $\begin{array}{l}\cdots \\
\cdots\end{array}$ & $\begin{array}{l}\cdots \\
\ldots\end{array}$ & $\begin{array}{l}\ldots \\
\ldots\end{array}$ & MAY97 \\
\hline HD 93403 & $\begin{array}{l}\text { O5.5 I } \\
\text { O7 V }\end{array}$ & $\begin{array}{l}>15.5 \\
>8.8\end{array}$ & $\begin{array}{l}39.3(11) \\
38.1(11)\end{array}$ & $\begin{array}{l}\cdots \\
\cdots\end{array}$ & $\begin{array}{l}\cdots \\
\cdots\end{array}$ & RAUO0 \\
\hline \multicolumn{7}{|c|}{ contact or first interaction } \\
\hline $\begin{array}{l}\text { Sk-67 } 67^{\circ} 105 \\
\text { (LMC) }\end{array}$ & $\begin{array}{l}\text { O4f } \\
\text { O6 V }\end{array}$ & $\begin{array}{l}47.2(8) \\
29.8(6)\end{array}$ & $\begin{array}{l}44.6(13) \\
41.0\end{array}$ & $\begin{array}{l}15.6(1) \\
12.2(3)\end{array}$ & $\begin{array}{l}\cdots \\
\cdots\end{array}$ & HAE94 \\
\hline $\begin{array}{l}\text { TU Mus } \\
\text { HD } 100213\end{array}$ & $\begin{array}{l}\mathrm{O} 8 \mathrm{~V} \\
\mathrm{O} 8 \mathrm{~V}\end{array}$ & $\begin{array}{l}17.2 \\
10.8\end{array}$ & $\begin{array}{l}\cdots \\
\cdots\end{array}$ & $\begin{array}{l}\cdots \\
\cdots\end{array}$ & $\begin{array}{l}258(26) \\
201(20)\end{array}$ & STI95b \\
\hline $\begin{array}{l}\text { V382 Cyg } \\
\text { HD 228854 }\end{array}$ & $\begin{array}{l}\text { O7 } \\
\cdots\end{array}$ & $\begin{array}{l}32.6(18) \\
22.9(13)\end{array}$ & $\begin{array}{l}37.6(18) \\
36.7(17)\end{array}$ & $\begin{array}{l}8.8(6) \\
7.4(6)\end{array}$ & $\begin{array}{l}\cdots \\
\cdots\end{array}$ & POP91 \\
\hline & $\begin{array}{l}\text { O7.3 V } \\
\text { O7.7 V }\end{array}$ & $\begin{array}{l}26.0(7) \\
19.3(4)\end{array}$ & $\begin{array}{l}36.0(5) \\
35.0(5)\end{array}$ & $\begin{array}{l}9.6(1) \\
8.4(1)\end{array}$ & $\begin{array}{l}258(3) \\
226(3)\end{array}$ & HAR97 \\
\hline & $\begin{array}{l}\text { O6.5 V((f)) } \\
\text { O6 V }((f))\end{array}$ & $\begin{array}{l}29.7(11) \\
20.3(9)\end{array}$ & $\begin{array}{l}41.2(6) \\
42.2(10)\end{array}$ & $\begin{array}{l}11.1(20) \\
9.2(17)\end{array}$ & $\begin{array}{l}\cdots \\
\cdots\end{array}$ & BUR97 \\
\hline \multicolumn{7}{|c|}{ hot Algol types } \\
\hline $\begin{array}{l}\mathrm{AzV} 73 \\
\text { (SMC) }\end{array}$ & $\begin{array}{l}\text { O8.5 V } \\
\text { B0 III }\end{array}$ & $\begin{array}{l}25.3(7) \\
22.0(8)\end{array}$ & $\begin{array}{l}37.0 \\
27.1\end{array}$ & $\begin{array}{l}11.5(5) \\
15.5(4)\end{array}$ & $\begin{array}{l}\cdots \\
\cdots\end{array}$ & OST01b \\
\hline $\begin{array}{l}\text { HV } 1620 \\
\text { (SMC) }\end{array}$ & $\begin{array}{l}\text { O9 V } \\
\text { O9.5 III }\end{array}$ & $\begin{array}{l}20 . \\
15 .\end{array}$ & $\begin{array}{l}33.0(45) \\
24.4(35)\end{array}$ & $\begin{array}{l}6 . \\
11 .\end{array}$ & $\begin{array}{l}\ldots \\
\cdots\end{array}$ & PRI98 \\
\hline $\begin{array}{l}\text { HV 2241 } \\
\text { (LMC) }\end{array}$ & $\begin{array}{l}\text { O7 III } \\
\text { B0 III }\end{array}$ & $\begin{array}{l}36.2(7) \\
18.4(7)\end{array}$ & $\begin{array}{l}39.9 \\
30.7\end{array}$ & $\begin{array}{l}14.9(4) \\
13.7(4)\end{array}$ & $\begin{array}{l}\ldots \\
\ldots\end{array}$ & OST01c \\
\hline $\begin{array}{l}\text { HV } 2543 \\
\text { (LMC) }\end{array}$ & $\begin{array}{l}\text { O8 } \\
\text { O9 }\end{array}$ & $\begin{array}{l}25.6(7) \\
15.6(10)\end{array}$ & $\begin{array}{l}34.7 \\
28.2\end{array}$ & $\begin{array}{l}15.5(4) \\
14.0(4)\end{array}$ & $\begin{array}{l}\ldots \\
\ldots\end{array}$ & OST00 \\
\hline \multirow[t]{2}{*}{$\begin{array}{l}\text { AO Cas } \\
\text { HD } 1337\end{array}$} & $\begin{array}{l}\text { O9.5 III } \\
\text { O8 V }\end{array}$ & $\begin{array}{l}12.4(17) \\
18.3(18)\end{array}$ & $\begin{array}{l}32.0 \\
36.0\end{array}$ & $\begin{array}{l}10.3(8) \\
7.9(23)\end{array}$ & $\begin{array}{l}\ldots \\
\ldots\end{array}$ & GIE91 \\
\hline & $\begin{array}{l}\text { O9.5 III } \\
\text { O8 V }\end{array}$ & $\begin{array}{l}>7.0(2) \\
>12.0(2)\end{array}$ & $\begin{array}{l}\ldots \\
\ldots\end{array}$ & $\begin{array}{l}\ldots \\
\ldots\end{array}$ & $\begin{array}{l}\ldots \\
\ldots\end{array}$ & STI97b \\
\hline $\begin{array}{l}\text { AB Cru } \\
\text { HD } 106871\end{array}$ & $\begin{array}{l}\mathrm{O} 8 \mathrm{~V} \\
\text { B0.5 }\end{array}$ & $\begin{array}{l}19.8(10) \\
7.0(7)\end{array}$ & $\begin{array}{l}35.8(5) \\
27.2(5)\end{array}$ & $\begin{array}{l}10.5(3) \\
8.9(3)\end{array}$ & $\begin{array}{l}\ldots \\
\ldots\end{array}$ & LOR94 \\
\hline $\begin{array}{l}\text { V961 Cen } \\
\text { HD } 115071\end{array}$ & $\begin{array}{l}\text { O9.5 V } \\
\text { B0.2 III }\end{array}$ & $\begin{array}{l}11.6(11) \\
6.7(7)\end{array}$ & $\begin{array}{l}32.0(20) \\
29.0(15)\end{array}$ & $\begin{array}{l}6.5(2) \\
7.2(2)\end{array}$ & $\begin{array}{l}134(13) \\
176(20)\end{array}$ & PEN02 \\
\hline HH Car & $\begin{array}{l}\text { O8 V } \\
\text { B0 III }\end{array}$ & $\begin{array}{l}17 . \\
14 .\end{array}$ & $\begin{array}{l}45 . \\
38 .\end{array}$ & $\begin{array}{l}6.1 \\
10.7\end{array}$ & $\begin{array}{l}\ldots \\
\ldots\end{array}$ & MAN85 \\
\hline $\begin{array}{l}\text { V448 Cyg } \\
\text { HD } 190967\end{array}$ & $\begin{array}{l}\text { O9.5 V } \\
\text { B1 II-Ib }\end{array}$ & $\begin{array}{l}25.2(7) \\
14.0(7)\end{array}$ & $\begin{array}{l}30.0(5) \\
20.5(6)\end{array}$ & $\begin{array}{l}6.7(2) \\
16.3(3)\end{array}$ & $\begin{array}{c}52(2) \\
127(2)\end{array}$ & HAR97 \\
\hline \multirow[t]{2}{*}{$\begin{array}{l}\text { LZ Cep } \\
\text { HD } 209481\end{array}$} & $\begin{array}{l}\text { O9 V } \\
\text { O9 V }\end{array}$ & $\begin{array}{l}18 .(4) \\
6.8(17)\end{array}$ & $\begin{array}{l}34.0 \\
34.0\end{array}$ & $\begin{array}{l}10.7(11) \\
7.8(8)\end{array}$ & $\begin{array}{l}205(13) \\
139(13)\end{array}$ & HOW91 \\
\hline & $\begin{array}{l}\text { O8.5 III } \\
\text { O9.5 V }\end{array}$ & $\begin{array}{l}15.1(4) \\
6.3(2)\end{array}$ & $\begin{array}{l}31.9(5) \\
30.1(5)\end{array}$ & $\begin{array}{l}9.0(2) \\
7.5(1)\end{array}$ & $\begin{array}{l}147(4) \\
125(1)\end{array}$ & HAR98b \\
\hline XZ Cep & $\begin{array}{l}\text { O9.5 V } \\
\text { B1 III }\end{array}$ & $\begin{array}{l}15.8(4) \\
6.4(3)\end{array}$ & $\begin{array}{l}30.0(5) \\
23.1(4)\end{array}$ & $\begin{array}{l}7.0(2) \\
10.5(3)\end{array}$ & $\begin{array}{c}69(2) \\
105(2)\end{array}$ & HAR97 \\
\hline
\end{tabular}


Table 3. (Cont'd.) Evolved systems (primary/secondary).

\begin{tabular}{|c|c|c|c|c|c|c|}
\hline $\begin{array}{l}\text { name/ } \\
\text { HD/other }\end{array}$ & $\begin{array}{l}\text { spectral } \\
\text { type }\end{array}$ & $\begin{array}{l}M \\
\left(\mathrm{M}_{\odot}\right)\end{array}$ & $\begin{array}{l}T_{\text {eff }} \\
(\mathrm{kK})\end{array}$ & $\begin{array}{l}R \\
\left(\mathrm{R}_{\odot}\right) \\
\end{array}$ & $\begin{array}{l}v_{\text {eq }} \\
\left(\mathrm{km} \mathrm{s}^{-1}\right)\end{array}$ & Ref. \\
\hline \multicolumn{7}{|c|}{ peculiar mass/luminosity systems } \\
\hline \multirow[t]{2}{*}{$\begin{array}{l}\text { SZ Cam } \\
\text { HD } 25638\end{array}$} & $\begin{array}{l}\text { O9.5 V } \\
\text { B0 }\end{array}$ & $\begin{array}{l}18.5(7) \\
16.1(6)\end{array}$ & $\begin{array}{l}29.7(5) \\
27.2(5)\end{array}$ & $\begin{array}{l}8.5(3) \\
7.9(3)\end{array}$ & $\begin{array}{l}158(5) \\
148(6)\end{array}$ & HAR98b \\
\hline & $\begin{array}{l}\text { O9 IV } \\
\text { B0.5 V }\end{array}$ & $\begin{array}{l}15.3(5) \\
10.8(4)\end{array}$ & $\begin{array}{l}33.0 \\
27.5(3)\end{array}$ & $\begin{array}{l}9.0(9) \\
6.6(7)\end{array}$ & $\begin{array}{l}\cdots \\
\cdots\end{array}$ & LOR98 \\
\hline $\begin{array}{l}\text { LY Aur } \\
\text { HD 35921 }\end{array}$ & $\begin{array}{l}\text { O9.5 III:n } \\
\text { B0 III }\end{array}$ & $\begin{array}{l}24.0(4) \\
12.7(3)\end{array}$ & $\begin{array}{l}32.1 \\
28.8\end{array}$ & $\begin{array}{l}\ldots \\
\ldots\end{array}$ & $\begin{array}{l}190 \\
170\end{array}$ & STI94b \\
\hline $\begin{array}{l}\delta \text { Ori A } \\
\text { HD } 36486\end{array}$ & $\begin{array}{l}\text { O9.5 II } \\
\text { B0.5 III }\end{array}$ & $\begin{array}{l}11.2(18) \\
5.6(8)\end{array}$ & $\begin{array}{l}33.0 \\
27.6\end{array}$ & $\begin{array}{l}\text { 13. (4) } \\
\text { 5. (2) }\end{array}$ & $\begin{array}{l}171(7) \\
150(17)\end{array}$ & HAR02 \\
\hline $\begin{array}{l}\text { ŁOri } \\
\text { HD } 37043\end{array}$ & $\begin{array}{l}\text { O9 III } \\
\text { B1 III }\end{array}$ & $\begin{array}{l}\text { 23. (10) } \\
\text { 13. (6) }\end{array}$ & $\begin{array}{l}32.5 \\
24.0\end{array}$ & $\begin{array}{l}8.3(20) \\
5.4(7)\end{array}$ & $\begin{array}{c}127(17) \\
81(11)\end{array}$ & $\begin{array}{l}\text { MAR00 } \\
\text { BAG01 }\end{array}$ \\
\hline $\begin{array}{l}\delta \mathrm{Cir} \\
\mathrm{HD} 135240\end{array}$ & $\begin{array}{l}\text { O7 III-V } \\
\text { O9.5 V }\end{array}$ & $\begin{array}{l}21.6(20) \\
12.4(10)\end{array}$ & $\begin{array}{l}37.5(15) \\
33.0(10)\end{array}$ & $\begin{array}{l}10.2(10) \\
6.4(7)\end{array}$ & $\begin{array}{l}156(10) \\
147(21)\end{array}$ & PEN01 \\
\hline \multicolumn{7}{|c|}{ supergiant systems } \\
\hline $\begin{array}{l}\text { Plaskett's } \\
\text { HD } 47129\end{array}$ & $\begin{array}{l}\text { O7.5 I } \\
\text { O6 I }\end{array}$ & $\begin{array}{l}>41 .(8) \\
>48 .(5)\end{array}$ & $\begin{array}{l}35.1 \\
38.4\end{array}$ & $\begin{array}{l}21.5 \\
18 .(4)\end{array}$ & $\begin{array}{l}78 \\
310\end{array}$ & $\begin{array}{l}\text { BAG92 } \\
\text { STI97b }\end{array}$ \\
\hline $\begin{array}{l}\text { UW CMa } \\
\text { HD } 57060\end{array}$ & $\begin{array}{l}\text { O7.5-8 Iab } \\
\text { O9.7 Ib }\end{array}$ & $\begin{array}{l}16 . \\
19 .\end{array}$ & $\begin{array}{l}33.8 \\
29.0\end{array}$ & $\begin{array}{l}13 . \\
10 .\end{array}$ & $\begin{array}{l}\ldots \\
\ldots\end{array}$ & $\begin{array}{l}\text { BAG94 } \\
\text { STI97b }\end{array}$ \\
\hline \multirow[t]{3}{*}{$\begin{array}{l}\text { V1007 Sco } \\
\text { HD } 152248\end{array}$} & $\begin{array}{l}\text { O7 I } \\
\text { O7 I }\end{array}$ & $\begin{array}{l}24.2(20) \\
25.8(20)\end{array}$ & $\begin{array}{l}35.0(15) \\
37.0(15)\end{array}$ & $\begin{array}{l}13.4(20) \\
12.9(20)\end{array}$ & $\begin{array}{l}171 \\
182\end{array}$ & PEN99 \\
\hline & $\begin{array}{l}\cdots \\
\ldots\end{array}$ & $\begin{array}{l}28.4 \\
29.5\end{array}$ & $\begin{array}{l}\cdots \\
\cdots\end{array}$ & $\begin{array}{l}15.6 \\
17.1\end{array}$ & $\begin{array}{l}\cdots \\
\cdots\end{array}$ & MAY01 \\
\hline & $\begin{array}{l}\text { O7.5 III(f) } \\
\text { O7 III(f) }\end{array}$ & $\begin{array}{l}29.6 \\
29.9\end{array}$ & $\begin{array}{l}37.1(10) \\
38.1(10)\end{array}$ & $\begin{array}{l}15.4(4) \\
14.9(4)\end{array}$ & $\begin{array}{l}\cdots \\
\cdots\end{array}$ & SAN01 \\
\hline HD 166734 & $\begin{array}{l}\text { O7.5 If } \\
\text { O9 I }\end{array}$ & $\begin{array}{l}>29 \\
>31\end{array}$ & $\begin{array}{l}\cdots \\
\cdots\end{array}$ & $\begin{array}{l}23 . \\
24 .\end{array}$ & $\begin{array}{l}\cdots \\
\cdots\end{array}$ & CON80 \\
\hline V729 Cyg & $\begin{array}{l}\text { O7 Ianfp } \\
\text { O6-7 Ia }\end{array}$ & $\begin{array}{l}\text { 47. (9) } \\
\text { 13. (3) }\end{array}$ & $\begin{array}{l}\cdots \\
\cdots\end{array}$ & $\begin{array}{l}\text { 31. (4) } \\
\text { 28. (5) }\end{array}$ & $\begin{array}{l}\cdots \\
\cdots\end{array}$ & RAU99 \\
\hline \multicolumn{7}{|c|}{ massive $X$-ray binaries } \\
\hline $\begin{array}{l}\text { LMCX-4 } \\
\text { (LMC) }\end{array}$ & $\begin{array}{l}\text { O7 III-V } \\
\text { NS }\end{array}$ & $\begin{array}{c}15.8(23) \\
1.5(4)\end{array}$ & $\begin{array}{l}\ldots \\
\ldots\end{array}$ & $\begin{array}{l}8.0(10) \\
\ldots\end{array}$ & $\begin{array}{l}188(50) \\
\ldots\end{array}$ & VAN95 \\
\hline Cen X-3 & $\begin{array}{l}\text { O6.5 II-III } \\
\text { NS }\end{array}$ & $\begin{array}{c}18.9(40) \\
1.1(5)\end{array}$ & $\begin{array}{l}\ldots \\
\ldots\end{array}$ & $\begin{array}{l}11.1(18) \\
\ldots\end{array}$ & $\begin{array}{l}274(30) \\
\ldots\end{array}$ & VAN95 \\
\hline
\end{tabular}

donor star. Most of the gainer stars have spectral subtypes later than O7 which suggests that either this stage is too short-lived or bypassed altogether in more massive systems. The fourth group is a diverse sample with unusually low masses or peculiar overluminosities that appear to implicate some past interaction. This group also includes the system $\iota$ Ori which may have swapped binary partners in a past binary-binary collision (Bagnuolo et al. 2001). The fifth group consists of pairs of supergiant stars in which mass transfer may be partially mitigated by colliding winds outflow. The final category consists of two X-ray binary systems for which the orbital motion was measured by pulsar timing. These diverse categories offer us a glimpse of the kinds of evolutionary processes that determine the fate of massive close binaries.

Acknowledgments. Support for this work was provided by NASA through grant numbers GO-8308 and GO-9449 from the Space Telescope Science Insti- 
tute, which is operated by the Association of Universities for Research in Astronomy, Inc., under NASA contract NAS5-26555.

\section{References}

Albacete Colombo, J.F., Morrell, N.I., Niemela, V.S., et al. 2001, MNRAS 326, 78 (ALB01)

Andersen, J. 1991, The A\&A Review 3, 91

Andersen, J., Clausen, J.V. 1989, A\&A 213, 183 (AND89)

Antokhina, E.A., Moffat, A.F.J., Antokhin, I.I., et al: 2000, ApJ 529, 463 (ANT00)

Bagnuolo, W.G., Jr., Gies, D.R., Hahula, M.E., et al. 1994, ApJ 423, 446 (BAG94)

Bagnuolo, W.G., Jr., Gies, D.R., Wiggs, M.S. 1992, ApJ 385, 708 (BAG92)

Bagnuolo, W.G., Jr., Riddle, R.L., Gies, D.R., et al. 2001, ApJ 554, 362 (BAG01)

Batten, A.H. 1968, AJ 73, 55

Bell, S.A., Hilditch, R.W., Adamson, A.J. 1987, MNRAS 225, 961 (BEL87)

Benvenuto, O.G., Serenelli, A.M., Althaus, L.G., et al. 2002, MNRAS 330, 435

Bertrand, J.-F., St-Louis, N., Moffat, A.F.J. 1998, in: I.D. Howarth (ed.), BoulderMunich II: Properties of Hot, Luminous Stars, ASP-CS 131, 376 (BER98)

Burkholder, V., Massey, P., Morrell, N. 1997, ApJ 490, 328 (BUR97)

Conti, P.S., Ebbets, D., Massey, P., Niemela, V.S. 1980, ApJ 238, 184 (CON80)

Figueiredo, J., De Greve, J.-P., Hilditch, R.W. 1994, A\&A 283, 144

Freyhammer, L.M., Clausen, J.V., Arentoft, T., et al. 2001, A\&A 369, 561 (FRE01)

Garcia, B., Mermilliod, J.C. 2001, A\&A 368, 122 (GAR01)

Gies, D.R., Penny, L.R., Mayer, P., Drechsel, H., Lorenz, R. 2002, ApJ 574, 957 (GIE02)

Gies, D.R., Wiggs, M.S. 1991, ApJ 375, 321 (GIE91)

Gies, D.R., Mason, B.D., Hartkopf, W.I., et al. 1993, AJ 106, 2072

Gies, D.R., Mason, B.D., Bagnuolo, W.G., Jr., et al. 1997, ApJ (Letters) 475, L49

Haefner, R., Simon, K.P., Fiedler, A. 1994, A\&A 288, L9 (HAE94)

Harries, T.J., Hilditch, R.W. 1998a, in: I.D. Howarth (ed.) Boulder-Munich II: Properties of Hot, Luminous Stars, ASP-CS 131, 401

Harries, T.J., Hilditch, R.W., Hill, G. 1997, MNRAS 285, 277 (HAR97)

Harries, T.J., Hilditch, R.W., Hill, G. 1998b, MNRAS 295, 386 (HAR98b)

Harvin, J.A., Gies, D.R., Bagnuolo, W.G., Jr., et al. 2002, ApJ 565, 1216 (HAR02)

Heger, A., Langer, N. 2000, ApJ 544, 1016

Herrero, A., Kudritzki, R.-P., Vílchez, J.M., et al. 1992, A\&A 261, 209

Herrero, A., Puls, J., Villamariz, M.R. 2000, A\&A 354, 193

Hilditch, R.W., Bell, S.A. 1987, MNRAS 229, 529

Hilditch, R.W., Harries, T.J., Bell, S.A. 1996, A\&A 314, 165 (HIL96)

Hill, G., Hilditch, R.W., Aikman, G.C.L., Khalesseh, B. 1994, A\&A 282, 455 (HIL94)

Hill, G., Holmgren, D.E. 1995, A\&A 297, 127 (HIL95)

Howarth, I.D., Stickland, D.J., Prinja, R.K., et al. 1991, The Observatory 111, 167 (HOW91)

Hutchings, J. B. 1975, PASP 87, 529

Lamontagne, R., Moffat, A.F.J., Drissen, L., et al. 1996, AJ 112, 2227

Lorenz, R., Mayer, P., Drechsel, H. 1994, A\&A 291, 185 (LOR94)

Lorenz, R., Mayer, P., Drechsel, H. 1998, A\&A 332, 909 (LOR98) 
Mandrini, C.H., Mendez, R.H., Ferrer, O.E., et al. 1985, RMxAA 11, 99 (MAN85)

Marchenko, S.V., et al. 2000, MNRAS 317, 333 (MAR00)

Martins, F., Schaerer, D., Hillier, D.J. 2002, A\&A 382, 999

Massey, P., Penny, L.R., Vukovich, J. 2002, ApJ 565, 982 (MAS02)

Mayer, P., Lorenz, R., Drechsel, H. 1997, A\&A 320, 109 (MAY97)

Mayer, P., Harmanec, P., Lorenz, R., Drechsel, H., Eenens, P., Corral, L.J., Morrell, N. 2001, in: D. Vanbeveren (ed.), The Influence of Binaries on Stellar Population Studies (Dordrecht: Kluwer), p. 567 (MAY01)

Meynet, G., Maeder, A. 2000, A\&A 361, 101

Morrell, N.I., Barbá, R.H., Niemela, V.S., et al. 2001, MNRAS 326, 85 (MOR01)

Ostrov, P.G. 2001a, MNRAS 321, L25 (OST01a)

Ostrov, P.G. 2001b, A\&A 380, 258 (OST01b)

Ostrov, P.G., Lapasset, E., Morrell, N.I. 2000, A\&A 356, 935 (OST00)

Ostrov, P.G., Morrell, N.I., Lapasset, E. 2001c, A\&A 377, 972 (OST01c)

Pachoulakis, I. 1996, MNRAS 280, 153 (PAC96)

Penny, L.R., Gies, D.R., Bagnuolo, W.G., Jr. 1997, ApJ 483, 439 (PEN97)

Penny, L.R., Gies, D.R., Bagnuolo, W.G., Jr. 1999, ApJ 518, 450 (PEN99)

Penny, L.R., Seyle, D., Gies, D.R., Harvin, J.A., Bagnuolo, W.G., Jr., Thaller, M.L., Fullerton, A.W., Kaper, L. 2001, ApJ 548, 889 (PEN01)

Penny, L.R., Gies, D.R., Wise, J.H., Stickland, D.J., Lloyd, C. 2002, ApJ 575, 1050 (PEN02)

Popper, D.M. 1980, Ann. Review Astron. Astrophys. 18, 115

Popper, D.M., Hill, G. 1991, AJ 101, 600 (POP91)

Pritchard, J.D., Tobin, W., Clark, M., Guinan, E.F. 1998, MNRAS 297, 278 (PRI98)

Rauw, G., Sana, H., Antokhin, I.I., et al. 2001, MNRAS 326, 1149 (RAU01)

Rauw, G., Sana, H., Gosset, E., Vreux, J.-M., et al. 2000, A\&A 360, 1003 (RAU00)

Rauw, G., Vreux, J.-M., Bohannan, B. 1999, ApJ 517, 416 (RAU99)

Ribas, I., Jordi, C., Torra, J., Giménez, Á. 2000, MNRAS 313, 99

Sana, H., Rauw, G., Gosset, E. 2001, A\&A 370, 121 (SAN01)

Schaerer, D., Meynet, G., Maeder, A., Schaller, G. 1993, A\&AS 98, 523

Schaller, G., Schaerer, D., Meynet, G., Maeder, A. 1992, A\&AS 96, 269

Schönberner, D., Harmanec, P. 1995, A\&A 294, 509

Schweickhardt, J., Schmutz, W., Stahl, O., Szeifert, Th., Wolf, B. 1999, A\&A 347, 127

Simon, K. P., Sturm, E., Fiedler, A. 1994, A\&A 292, 507 (SIM94)

Stickland, D.J. 1995a, The Obeorvatory 115, 180 (STI95a)

Stickland, D.J. 1997b, The Observatory 117, 37 (STI97b)

Stickland, D.J., Koch, R.H., Pachoulakis, I., et al. 1994b, The Observatory 114, 107 (STI94b)

Stickland, D.J., Lloyd, C. 2001, The Observatory 121, 1 (STI01)

Stickland, D.J., Lloyd, C., Corcoran, M.F. 1994a, The Observatory 114, 284 (STI94a)

Stickland, D.J., Lloyd, C., Koch, R.H., Pachoulakis, I. 1995b, The Observatory 115, 317 (STI95b)

Stickland, D.J., Lloyd, C., Penny, L.R. 1997a, The Observatory 117, 213 (STI97a)

Sturm, E., Simon, K.P. 1994, A\&A 282, 93 (STU94)

Thackeray, A.D. 1966, MNRAS 134, 97 (THA66) 
Vanbeveren, D., van Rensbergen, W., de Loore, C. 1998, The Brightest Binaries (Dordrecht: Kluwer)

van der Hucht, K.A. 2001, New Astron. Reviews 45, 135

van Kerkwijk, M.H., van Paradijs, J., Zuiderwijk, E.J. 1995, A\&A 303, 497 (VAN95)

Vaz, L.P.R., Cunha, N.C.S., Vieira, E.F., et al. 1997, A\&A 327, 1094 (VAZ97)

\section{Discussion}

KAPER: You showed that hot Algols are overluminous for their mass. In high-mass $\mathrm{X}$-ray binaries, which have undergone a hot Algol phase and are presently in or near to their second phase of mass transfer, the systems experiencing Roche-lobe overflow also have a OB I companion which is luminous (e.g., Cen X-3, LMC X-4). However, those HMXBs which are just about to start Roche-lobe overflow (wind-fed systems like Vela X-1, 4U 1170-37) do not seem to be overluminous for their mass (Kaper 2001). How can we reconcile this with a previous hot Algol phase?

GIES: The visible stars in HMXBs represent the mass gainers during the earlier, hot Algol phase. The masses of these gainers are often consistent with the luminosities and spectral types of single stars with similar mass. It is possible that some of these gainers are re-jeuvenated by mass transfer and have assumed the luminosity appropriate for their new mass. Then, when they evolve to larger radii in the HMXB phase, their masses will be consistent with single star luminosities, at least until they suffer large mass loss.

WALBORN: How was the inclination of $\delta$ Ori A determined, and what are the uncertainties of the low derived masses?

GIES: The inclination was derived by fitting the partially eclipsing light curve. There is a range in the acceptable radii which can be adopted in the light curve analysis, and this leads to mass estimates of $11.2 \pm 1.8 \mathrm{M}_{\odot}$ and $5.6 \pm 0.8 \mathrm{M}_{\odot}$ for the primary and secondary, respectively. Thus, both stars are very overluminous for their masses.

CherepashchuK: Could you explain the definition of the luminosity of a rapidly rotating star? A rapidly rotationally star has a complicated temperature distribution due to gravitational darkening. How do you calculate the luminosity of such a star? Do you have some information about $v \sin i$ for investigated stars?

GIES: Luminosity is still defined by the energy emitted over the entire surface (even though the temperature varies from hot to cool from the pole to the equator). Ideally, we need to measure the projected rotational velocity, radii, and temperatures in a way that accounts for rotational distortion effects and our orientation to the system. This will require profile-fitting techniques that have not yet been widely adopted.

KUDRITZKI: It is interesting to see that the mass-discrepancy for O-type MS objects has been resolved. But what is the situation for supergiants?

GIES: Unfortunately, there are only a few supergiant systems available for investigation. The LMC binaries MACHO*05:34:41.3-69:31:39 (O3 If* primary) and Sk-67 ${ }^{\circ} 105$ (O4f) have masses that agree with single star evolutionary tracks. Both of these are probably systems in a stage before large scale mass transfer. The remaining systems containing supergiants are interacting binaries, and the supergiant masses are generally lower than the predicted masses for single stars. 
MAíz-ApellánIZ: Hoogerwerf et al. (2000, ApJ 544, L133) showed recently that $i$ Ori has indeed been a victim of a dynamical ejection, as you suggested when you mentioned that the two components could not be coeval. They used Hipparcos data to trace back the trajectories of two runaway stars to the point where $i$ Ori was $2 \mathrm{Myr}$ ago.

GIEs: The proper motions given by Hoogerwerf et al. do indeed show that the runaway stars AE Aur and $\mu \mathrm{Col}$ were coincident with $i$ Ori some 2 million years ago. This evidence, combined with the apparent no-coeval ages of the binary components and the large orbital eccentricity of the binary all indicate, that a binary-binary collision occurred that led to an exchange of binary components and the ejection of runaway stars.

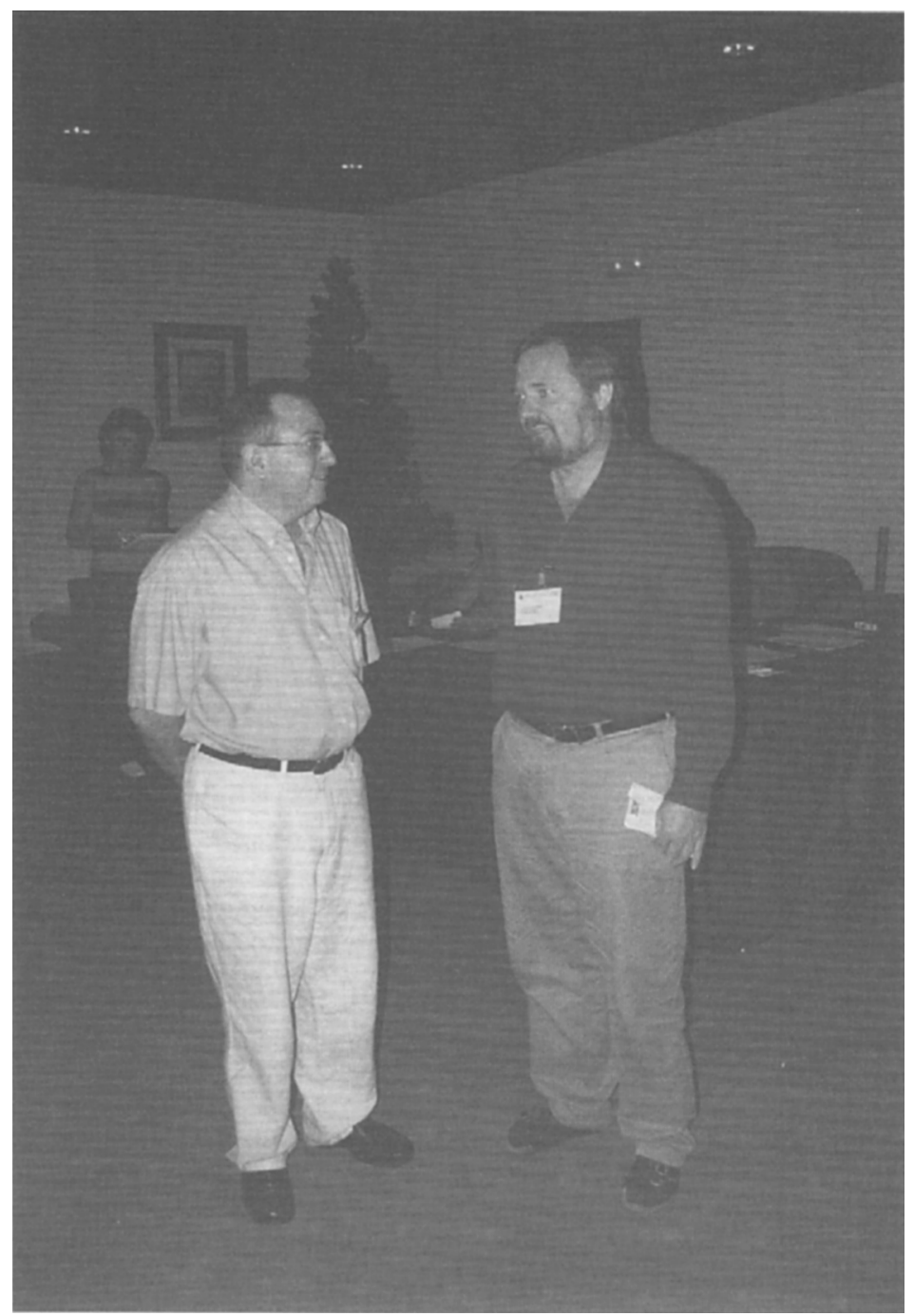

Artemio Herrero and Norbert Langer, contemplating massive binaries 